\title{
Investigation of hot carrier thermalization mechanisms in quantum well structures
}

\author{
Hamidreza Esmaielpour a ${ }^{\mathrm{a}}$, Laurent Lombez ${ }^{\mathrm{a}, \mathrm{b}}$, Maxime Giteau ${ }^{\mathrm{c}}$, Amaury Delamarre ${ }^{\mathrm{c}, \mathrm{d}}$, Daniel Ory ${ }^{\mathrm{e}}$, \\ Andrea Cattoni ${ }^{\mathrm{a}, \mathrm{d}}$, Stéphane Collin ${ }^{\mathrm{a}, \mathrm{d}}$, Jean-François Guillemoles ${ }^{\mathrm{a}, \mathrm{f}}$, and Daniel Suchet ${ }^{\mathrm{a}, \mathrm{f}}$ \\ ${ }^{a}$ CNRS-Institute Photovoltaïque d'Ile de France (IPVF), UMR IPVF 9006, Palaiseau, France; ${ }^{\mathrm{b}}$ \\ Laboratoire de Physique et Chimie des Nano-objets (LPCNO-INSA), Toulouse, France; ${ }^{c}$ NextPV, \\ LIA, Research Center for Advanced Science and Technology (RCAST), University of Tokyo, \\ Tokyo, Japan; ${ }^{\mathrm{d}}$ Centre de Nanosciences et de Nanotechnologies (C2N), CNRS, Université Paris- \\ Saclay, Palaiseau, France; ${ }^{\mathrm{e}}$ Électricité de France (EDF) R\&D, Palaiseau, France; ${ }^{\mathrm{f}}$ CNRS-Ecole \\ Polytechnique, UMR IPVF 9006, Palaiseau, France.
}

\begin{abstract}
In photovoltaic devices, thermalization of hot carriers generated by high energy photons is one of the major loss mechanisms, which limits the power conversion efficiency of solar cells. Hot carrier solar cells are proposed to increase the efficiency of this technology by suppressing phonon-mediated thermalization channels and extracting hot carriers isentropically. Therefore, designing hot carrier absorbers, which can inhibit electron-phonon interactions and provide conditions for the re-absorption of the energy of non-equilibrium phonons by (hot) carriers, is of significant importance in such devices. As a result, it is essential to understand hot carrier relaxation mechanisms via phonon-mediated pathways in the system. In this work, the properties of photo-generated hot carriers in an InGaAs multi-quantum well structure are studied via steady-state photoluminescence spectroscopy at various lattice temperatures and excitation powers. It is observed that by considering the contribution of thermalized power above the absorber band edge, it is possible to evaluate hot carrier thermalization mechanisms via determining the thermalization coefficient of the sample. It is seen that at lower lattice temperatures, the temperature difference between hot carriers and the lattice reduces, which is consistent with the increase of the quasi-Fermi level splitting for a given thermalized power at lower lattice temperatures. Finally, the spectral linewidth broadening of multiple optical transitions in the QW structure as a function of the thermalized power is investigated.
\end{abstract}

Keywords: Photoluminescence, hot carrier, thermalization mechanism, quasi-Fermi level splitting, spectral linewidth broadening.

\section{INTRODUCTION}

Hot carrier solar cells have attracted a lot of interest in recent years due to their potential to increase the efficiency of photovoltaic devices above the Shockley-Queisser limit (33\%) for single junction solar cells. ${ }^{12}$ Thermalization of hot carriers in solar cell absorbers is one of the major loss mechanisms, which limits the efficiency of photovoltaic devices. ${ }^{3}$ ${ }^{4}$ Therefore, by understanding the origin of thermalization loss and inhibiting heat dissipation, it is possible to improve the efficiency of solar cells.

In polar semiconductors, the interaction of hot carriers with longitudinal optical phonons (Fröhlich interactions) is one of the main relaxation pathways for hot carriers. ${ }^{56}$ These interactions are usually followed by the emission of low energy phonons via Klemens ${ }^{7}$ and Ridley ${ }^{8}$ mechanisms, which will finally lead to energy dissipation in the system. Therefore, by suppressing these relaxation pathways, or by reabsorbing the transferred energy to phonons, it is possible to increase the relaxation life time of hot carriers and to inhibit their thermalization loss. 91011

In this work, hot carrier properties and their thermalization mechanisms in InGaAs multi-quantum well (MQW) structure are investigated. This QW structure has already shown evidence of hot carrier effects, therefore, it is a good candidate to be applied for further understanding the characteristics of hot carriers in the system. ${ }^{12} 1314$ The InGaAs QW can slow down the thermalization mechanism, which is significant for observing hot carrier effects via photoluminescence (PL) spectroscopy. Furthermore, the quasi-Fermi level splitting of the photo-generated hot carriers at various lattice 
temperatures are also studied, which are applicable for the electrical characterizations of such a design. Finally, the spectral linewidth broadening of multiple optical transitions from the discrete energy levels in the QW structure is investigated.

\section{EXPERIMENTAL RESULTS AND DISCUSSIONS}

The thermalization effect of hot carriers is studied in InGaAs MQW structure, which consists of five un-doped InGaAs QWs $(5.5 \mathrm{~nm})$ and InGaAsP barriers $(10 \mathrm{~nm})$. To improve the accumulation of photo-generated carriers and to enhance the effects of hot carriers, wide gap InP cladding layers are designed to isolate the active region of the QW structure.

The hot carrier behavior is studied via steady-state photoluminescence spectroscopy at various lattice temperatures and excitation powers. The emitted PL spectra are registered by a hyperspectral luminescence imager, which creates spectrally and spatially resolved PL maps. The absolute photon flux emitted by the InGaAs MQW is determined via applying calibration methods. A continuous wave laser with an energy of $3.06 \mathrm{eV}$ (or $405 \mathrm{~nm}$ ) is used to excite electrons from the valence band into the conduction band. The fraction of the incident power absorbed within the QW region is determined by the transfer matrix analysis.

The thermodynamic properties of photo-generated hot carriers (the temperature, " $T$ ", and the quasi-Fermi level splitting, " $\Delta \mu ")$ are found via fitting the full PL spectrum with the generalized Planck's radiation law, as described by: 615

$$
I_{P L}(E)=\frac{2 \pi A(E) E^{2}}{h^{3} c^{2}}\left[\exp \left(\frac{E-\Delta \mu}{k_{B} T}\right)-1\right]^{-1},
$$

where " $A(E)$ " is the absorptivity of the sample, " $h$ " the Planck constant, " $k_{B}$ " the Boltzmann constant, " $c$ " the speed of light, and " $I_{P L}$ " the emitted PL intensity. To fit the full PL spectrum, it is required to determine the absorptivity of the sample via considering various optical transitions from discrete energy levels in the QW structure and the band-filling effect as a function of the excitation power. The detail of the full spectrum fit and its comparison with various fitting methods are described elsewhere. 131416

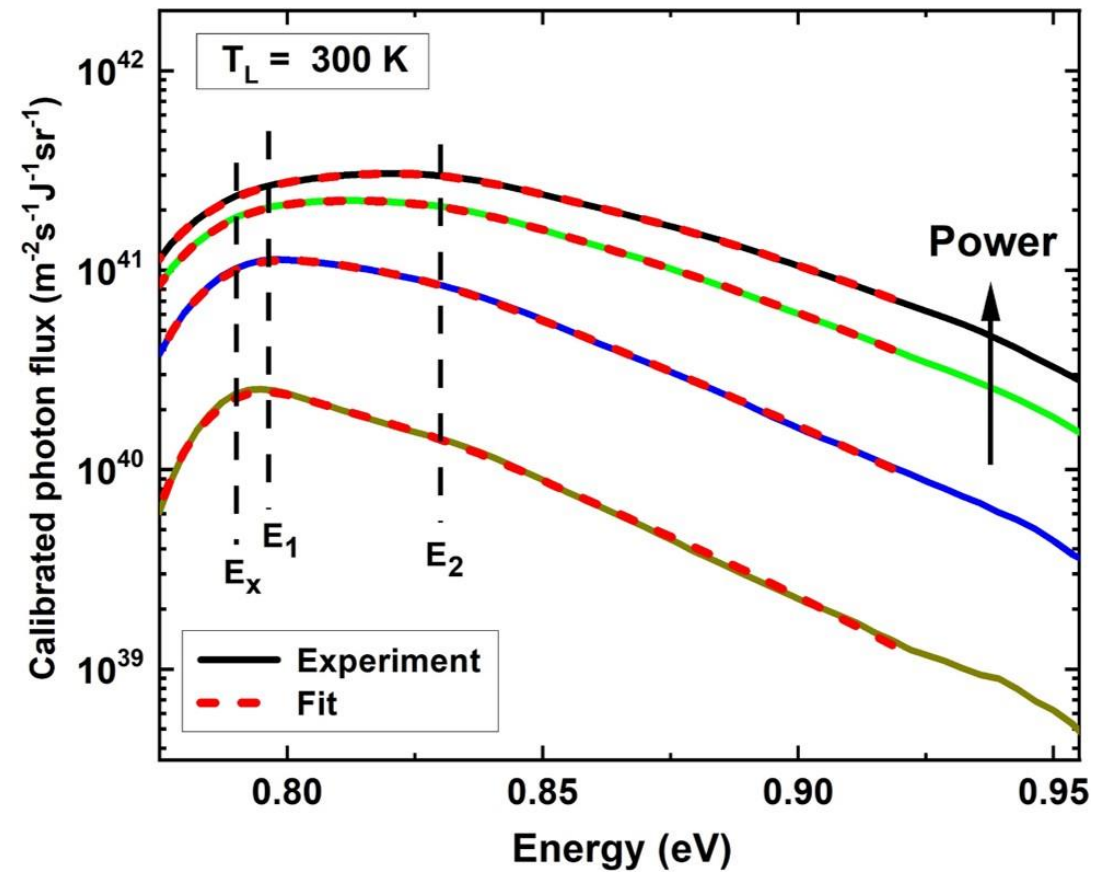

Figure 1. Excitation power-dependent PL spectra emitted by the InGaAs MQW structure at $300 \mathrm{~K}$. The dashed red lines show the results of the full PL spectrum fit. The positions of optical transitions in the QW structure are shown in the graph: $E_{x}, E_{1}$, and $E_{2}$ are the energy levels for the excitonic, the first and the second band-to-band transitions. 
By applying the full spectrum fit, three dominant optical transitions are found in the system: one excitonic and two bandto-band transitions, which are labeled by "x", "1", and "2", respectively. Figure 1 shows the PL spectra emitted by the QW structure at $300 \mathrm{~K}$ under various excitation powers. The results of the curve fitting are represented by dashed red lines for each individual spectrum in Figure 1.

The hot carrier temperature difference ( $\Delta T$ : the carrier temperature minus the lattice temperature) as a function of the absorbed power density $\left(P_{A b s .}\right)$ at two lattice temperatures $(300 \mathrm{~K}$ and $230 \mathrm{~K})$ are plotted in Figure 2 (a). The absorbed power density is determined by multiplying the excitation power and the absorbance of the QW structure in the active region (found by transfer matrix analysis) divided by the size of the laser spot (determined from hyperspectral images). In Figure 2 (a), it is observed that the effects of hot carriers become stronger at higher absorbed powers. In addition, the comparison between $\Delta T$ at two lattice temperatures indicates that the temperature of hot carriers reduces at the lower lattice temperature.

To evaluate the performance of the QW structure in inhibiting the thermalization of hot carriers, it is required to determine the amount of the thermalized power $\left(P_{t h}\right)$ above the band edge of the semiconductor, which can be approximated to a first order by: ${ }^{17}$

$$
P_{\text {th }} \approx \frac{E_{\text {laser }}-E_{\text {gap }}}{E_{\text {laser }}} P_{\text {abs }},
$$

where " $E_{\text {laser }}$ " and " $E_{\text {gap }}$ " are respectively the energy of the excitation (or laser) photons and the band gap of the absorber. The thermalization coefficient $(Q)$ of the sample can then be determined by: ${ }^{17} 18$

$$
P_{t h}=Q \cdot \Delta T
$$

The thermalization coefficient describes the efficiency of phonon-mediated pathways to thermalize hot carriers. Figure 2 (b) shows a linear plot of $\Delta T$ versus the thermalized power at two lattice temperatures. A linear relationship between the change in $\Delta T$ and $P_{t h}$ is observed, especially at high illuminations, where the density of non-equilibrium optical phonons is large. The slope of the plot in Figure 2 (b), as indicated by Equation 3, is inversely proportional with the Q-factor. The values determined for the thermalization coefficient at $300 \mathrm{~K}$ and $230 \mathrm{~K}$ are $12 \mathrm{~W} \cdot \mathrm{cm}^{-2} \cdot \mathrm{K}^{-1}$ and $15 \mathrm{~W} . \mathrm{cm}^{-2} . \mathrm{K}^{-1}$, respectively.
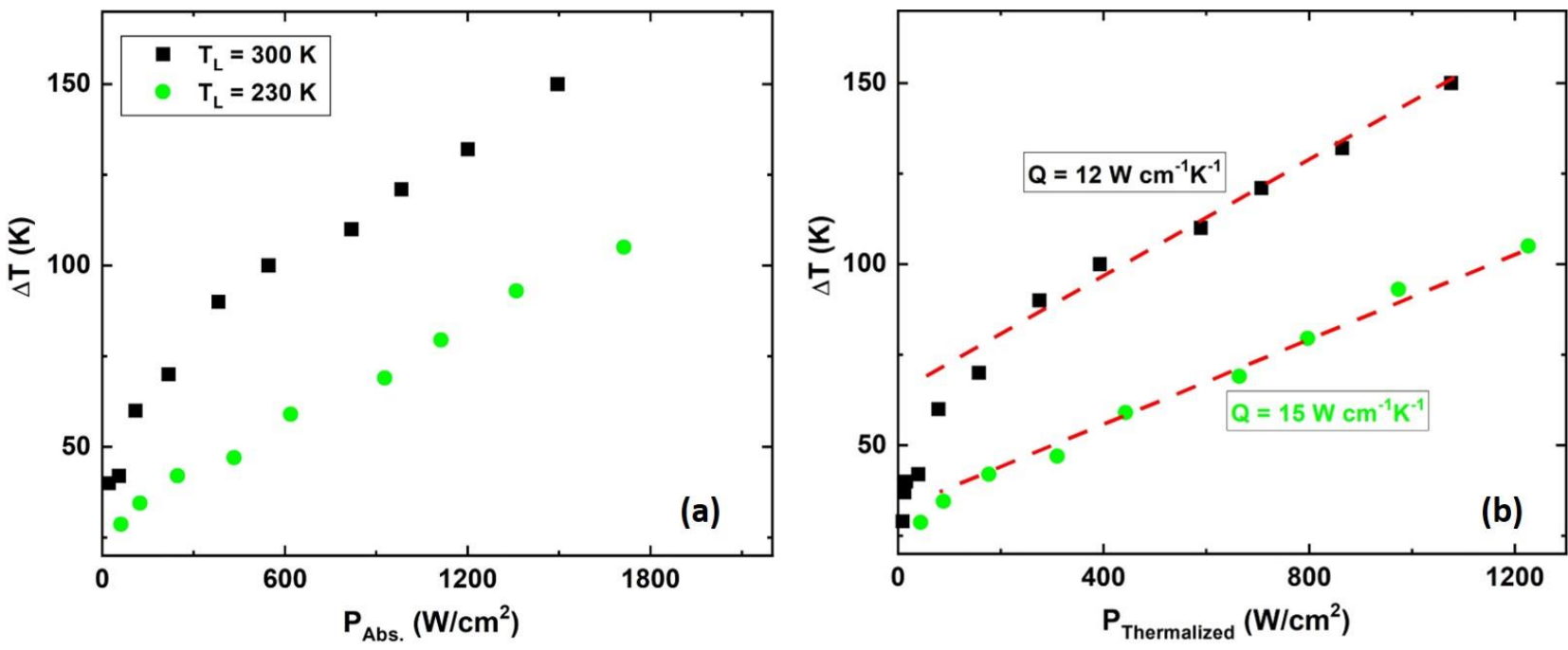

Figure 2. Carrier temperature difference $(\Delta T)$ as a function of (a) the absorbed power density, and (b) the thermalized power at $300 \mathrm{~K}$ (black) and $230 \mathrm{~K}$ (green). The dashed red lines show the linear fitting results, which are inversely proportional with the thermalization coefficient. 
The thermalization effect of hot carriers in the QW structure has also been investigated under lower energy excitation photons $(980 \mathrm{~nm})$. Similar thermalization coefficients are determined for the two laser excitations $(405 \mathrm{~nm}$ and $980 \mathrm{~nm})$, even though the energy transferred to hot carriers per incident photon is different. This effect indicates that by considering the amount of the thermalized power above the absorber band edge, it is possible to define a thermalization coefficient, which evaluates the performance of the sample in inhibiting the thermalization loss and is not dependent on the excitation wavelength. Similar behavior has been recently observed by Giteau et al. ${ }^{17}$ in thin GaAs absorbers and its origin is discussed in detail.

The results of $\Delta \mu$ for the hot carriers in the QW structure at various lattice temperatures are plotted in Figure 3 as a function of the thermalized power. It is seen that $\Delta \mu$ increases at higher excitation powers, which is associated with the increase in the density of photo-generated carriers in the system. In addition, $\Delta \mu$ increases at lower lattice temperatures, which is due to the lower rates of non-radiative recombination and widening of the absorber band gap. ${ }^{19}$ Moreover, the increase of $\Delta \mu$ at lower lattice temperatures is also due to the reciprocal relationship between the temperature of hot carriers and $\Delta \mu$. In other words, for a given density of photo-generated carriers, when the carrier temperature reduces, $\Delta \mu$ increases. $^{20}$

The position of the band gap energies at various lattice temperatures, which are determined from the PL spectrum, are indicated by dashed lines in Figure 3. It is seen that the energy separation between the band gap energies and the values of $\Delta \mu$ at high excitation powers, where $\Delta \mu$ flattens, increases at higher lattice temperatures. The origin of this effect can be due to the increase of non-radiative recombination and stronger hot carrier effects at higher lattice temperatures. ${ }^{19}$

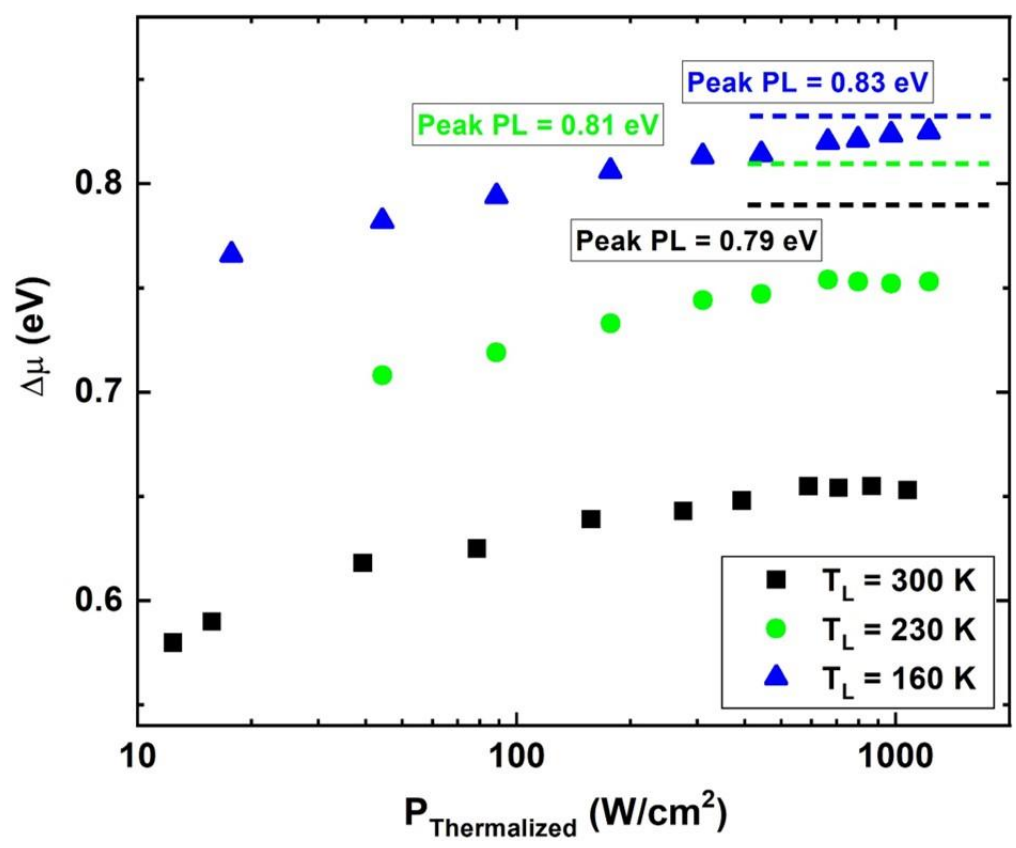

Figure 3. The quasi-Fermi level splitting $(\Delta \mu)$ as a function of the thermalized power at $300 \mathrm{~K}$ (black), $230 \mathrm{~K}$ (green), and $160 \mathrm{~K}$ (blue). The dashed lines indicate the band gap energies at various lattice temperatures, which are determined from the peak position of the PL spectrum.

The normalized PL spectra at room temperature under various excitation powers are plotted in Figure 4 (a). It is seen that by increasing the excitation power, there is an increase in the linewidth broadening of the PL spectra in both low and high energy sides. The broadening of the PL spectra at the high energy side is due to the combination of the effects of hot carriers, occupation of excited states, and charge scattering with phonons. ${ }^{13}{ }^{21}$ However, the broadening of the PL spectra on the low energy side is mainly due to the band-filling effect and the phonon-mediated charge scattering, the impacts of which can be determined from the absorptivity of the QW structure. ${ }^{16} 21$

To investigate the impact of non-equilibrium LO-phonons (or hot phonons) on the broadening of the PL spectrum, the linewidth broadening for various optical transitions in the QW structure as a function of the thermalized power is plotted in Figure 4 (b). It is seen that the linewidth broadening for the excitonic (blue) and the first band-to-band (pink) transitions 
increases as a function of power, however, the second band-to-band (gray) transition remains constant. The increase of the linewidth broadening for the excitonic transition as a function of power is due to their dissociation mechanisms at higher illuminations, which is in good agreement with the drop of their absorption amplitude at higher powers (it is not shown here). 2223
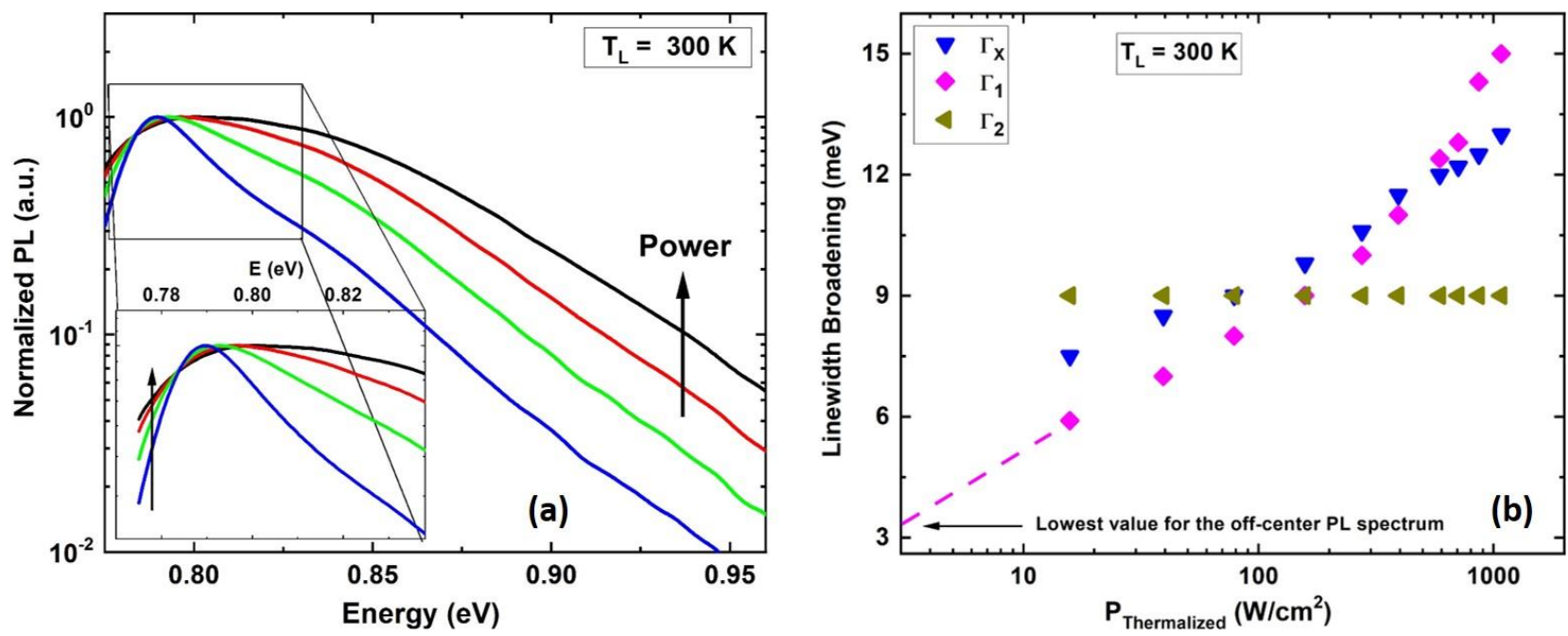

Figure 4. (a) Normalized PL spectra at $300 \mathrm{~K}$ under various excitation powers. The inset shows the region of the spectrum near the peak position. (b) The spectral linewidth broadening for various optical transitions as a function of the thermalized power at $300 \mathrm{~K}$. The values are determined via applying the full PL spectrum fit. $\Gamma_{x}, \Gamma_{1}$, and $\Gamma_{2}$ are respectively the linewidth broadening for the excitonic, the first, and the second band-to-band transitions in the QW structure.

In semiconductors, the change of the spectral linewidth $\left(\Gamma_{\text {tot }}\right)$ broadening as a function of the lattice temperature can be described by: 24

$$
\Gamma_{\text {tot }}(\mathrm{T})=\Gamma_{0}+\mathrm{K}_{\mathrm{LA}} \mathrm{T}+\frac{\gamma_{\mathrm{LO}}}{\left[\exp \left(\frac{\hbar \omega_{\mathrm{LO}}}{\mathrm{k}_{\mathrm{B}} \mathrm{T}}\right)-1\right]}+\Gamma_{\text {Imp. }} \exp \left(-\frac{\mathrm{E}_{\mathrm{b}}}{\mathrm{k}_{\mathrm{B}} \mathrm{T}}\right)
$$

where " $\Gamma_{0} "$ is due to the inhomogeneous broadening at $0 \mathrm{~K}$, the second term is due to the linewidth broadening due to charge interactions with acoustic phonons, the third term indicates the change in the linewidth due to carrier-LO phonon interactions ( $\hbar \omega_{\mathrm{LO}}$ " is the energy of LO-phonons and " $\gamma_{\mathrm{LO}}$ " is the Fröhlich coupling constant), and the last term is due to

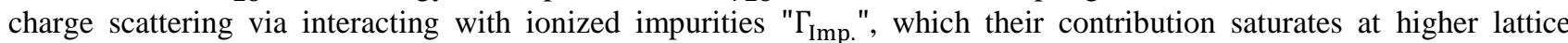
temperatures (" $\mathrm{E}_{\mathrm{b}}$ " is the energy of the ionized impurities). ${ }^{24}$

In polar (group III-V) semiconductors, the influence of longitudinal optical (LO) phonons on the broadening of the PL linewidth (the third term in Equation 4) at elevated lattice temperatures is significant. ${ }^{21}{ }^{24}$ Therefore, at such condition it is possible to correlate the PL linewidth broadening with the density of LO-phonons $\left(N_{L O}\right)$, as given by:

$$
\Gamma_{\text {high temperature }}(\mathrm{T}) \approx \gamma_{\mathrm{LO}} \cdot \mathrm{N}_{\mathrm{LO}} \text {. }
$$

The Fröhlich constant determines the strength of the interactions between hot carriers and LO-phonons in the absorber. This parameter remains constant at various lattice temperatures and excitation powers as long as the matrix element of the transition does not change. ${ }^{25}$ Therefore, it is possible to determine the Fröhlich constant for the first band-to-band transition from the linewidth broadening of the PL spectrum emitted by carriers in thermal equilibrium with the lattice (3.5 meV). The value determined for the Fröhlich constant is $\sim 9 \mathrm{meV}$, which is in good agreement with reported values for a such QW structure in literature. ${ }^{26}$ 
To correlate the spectral linewidth broadening with the density of non-equilibrium LO-phonons, it is assumed that the LOphonons are in thermal equilibrium with the hot carriers $\left(T_{C}=T_{L O}\right)$. Through this analysis, the calculated values for the spectral linewidth broadening are found to be smaller than the experimental results. This mismatch may indicate that it is required to consider other parameters to determine the density of non-equilibrium LO-phonons in the QW structure, or other mechanisms, such as charge screening, are also involved, which may have significant contributions in the broadening of the emitted PL spectra.

\section{CONCLUSION}

In this work, the properties of photo-generated hot carriers in the InGaAs MQW structure are investigated via PL spectroscopy. It is observed that the QW structure can limit the thermalization of hot carriers and by increasing the excitation power, the effects of hot carriers become stronger. The thermalization coefficient of the QW structure is determined via considering the fraction of the incident power thermalized above the absorber band edge. The thermalization coefficients of the sample at two lattice temperatures show similar values, although $\Delta T$ at a given thermalized power is less at the lower lattice temperature $(230 \mathrm{~K})$. The $\Delta \mu$ of hot carriers is determined as a function of the thermalized power, and exhibits larger values at higher illumination intensities. In addition, $\Delta \mu$ increases at lower lattice temperatures, which is in good agreement with lower rates of non-radiative recombination, greater bandgap energies, and smaller $\Delta T$ at lower lattice temperatures. Finally, the spectral linewidth broadening for various optical transitions in the QW structure is determined as a function of the thermalized power and the origin of their power-dependent behavior is investigated.

\section{ACKNOWLEDGMENT}

This work is performed in the framework of program 6: PROOF at IPVF (Proof of concept for PV innovation breakthrough). The authors would like acknowledge the financial support through the French ANR project, ICEMAN (ANR-19-CE05-0019), for hot carrier solar cell investigations.

\section{REFERENCES}

[1] Ross, R. T., and Nozik, A. J. "Efficiency of hot-carrier solar energy converters." Journal of Applied Physics 53.5 (1982): 3813-3818.

[2] Green, M. A. "Third generation photovoltaics." (2006).

[3] Conibeer, G. et al. Progress on hot carrier cells. Sol. Energy Mater. Sol. Cells 93, 713-719 (2009).

[4] Guillemoles, J. F., et al. "Guide for the perplexed to the Shockley-Queisser model for solar cells." Nature photonics 13.8 (2019): 501-505.

[5] Lyon, S. A. "Spectroscopy of hot carriers in semiconductors." Journal of luminescence 35.3 (1986): 121-154.

[6] Shah, J. "Hot carriers in quasi-2-D polar semiconductors." IEEE journal of quantum electronics 22.9 (1986): 1728 1743.

[7] Klemens, P. G., "Anharmonic decay of optical phonons," Physical Review 148.2 (1966): 845.

[8] Ridley, B. K., "The LO phonon lifetime in GaN," Journal of Physics: Condensed Matter 8.37 (1996): L511.

[9] Conibeer, G. et al. "Hot carrier solar cell absorber prerequisites and candidate material systems." Solar Energy Materials and Solar Cells 135 (2015): 124-129.

[10] König, D., et al. "Non-equilibrium dynamics, materials and structures for hot carrier solar cells: a detailed review," Semiconductor Science and Technology 35, no. 7 (2020): 073002.

[11] Esmaielour, H., et al. "Enhanced hot carrier lifetimes in quantum wells with inhibited phonon coupling," Sceintific Reports 8 (2018): 12473.

[12] Rodière, J., et al. "Experimental evidence of hot carriers solar cell operation in multi-quantum wells heterostructures." Applied Physics Letters 106.18 (2015): 183901.

[13] Gibelli, F., et al. "Two carrier temperatures non-equilibrium generalized Planck law for semiconductors." Physica B: Condensed Matter 498 (2016): 7-14. 
[14] Esmaielpour, H., et al. "Investigation of the spatial distribution of hot carriers in quantum-well structures via hyperspectral luminescence imaging," Journal of Applied Physics 128.16 (2020): 165704.

[15] Wurfel, P., "The chemical potential of radiation,” J. Phys. C: Solid State Phys. 15, 3967 (1982).

[16] Nguyen, D. T., et al. "Quantitative experimental assessment of hot carrier-enhanced solar cells at room temperature," Nature Energy 3.3 (2018): 236-242.

[17] Giteau, M., et al. "Identification of surface and volume hot-carrier thermalization mechanisms in ultrathin GaAs layers," Journal of Applied Physics 128.19 (2020): 193102.

[18] Le Bris, A., et al. "Thermalisation rate study of GaSb-based heterostructures by continuous wave photoluminescence and their potential as hot carrier solar cell absorbers," Energy \& Environmental Science. 2012;5(3):6225-32.

[19] Kluftinger, B., et al. "Temperature-dependent study of the quasi-Fermi level separation in double quantum well PIN structures." Microelectronic Engineering 51 (2000): 265-274.

[20] Nelson, J. A., "The physics of solar cells." World Scientific Publishing Company, 2003.

[21] Esmaielpour, H., et al. "Effect of occupation of the excited states and phonon broadening on the determination of the hot carrier temperature from continuous wave photoluminescence in InGaAsP quantum well absorbers." Progress in Photovoltaics: Research and Applications 25.9 (2017): 782-790.

[22] Eccleston, R., et al. "Density-dependent exciton radiative lifetimes in GaAs quantum wells." Physical Review B 45.19 (1992): 11403.

[23] Chuang, S. L. "Physics of optoelectronic devices." Wiley New York, 1995.

[24] Lee, J., et al. "Luminescence linewidths of excitons in GaAs quantum wells below 150 K." Physical Review B 33.8 (1986): 5512.

[25] Spector, H. N., et al. "Exciton linewidth in semiconducting quantum-well structures." Physical Review B 34.4 (1986): 2554.

[26] Shen, W. Z., "Study of exciton-longitudinal optical phonon coupling in quantum wells for optoelectronic applications." Applied Physics Letters 79.9 (2001): 1285-1287. 